\title{
12 Prevention of suicidal behavior in adolescents with post-traumatic stress disorder
}

\author{
Eugene Ruby and Leo Sher
}

Post-traumatic stress disorder (PTSD) is significantly associated with an increased risk for suicidal behavior among adolescents. Suicide is one of the top three causes of adolescent deaths worldwide. Despite the strong relationship between PTSD and suicidal behavior, precise causal pathways linking PTSD to suicide in adolescents remains unclear. A slew of mediating factors and variables commonly present themselves with both suicide and PTSD, including comorbid psychiatric disorders, exposure to different forms of trauma and stressful life events, core neurobiological changes, and mental, emotional, and physiological states like hyperarousal, impulsivity, and aggression. Because youth is such a critical stage of development, it is very important that at-risk adolescents are identified and referred for treatment. With many treatment challenges in these populations, effective implementation and use of prevention methods are of increasing importance. The most proven prevention methods include physician education, means restriction, and gatekeeper training. Other strategies that have received empirical support are public education campaigns and implementing guidelines for the media, including those for television, print media, and the Internet.

\subsection{Introduction}

A 2010 report, using the National Comorbidity Survey-Adolescent Supplement, found the lifetime prevalence of post-traumatic stress disorder (PTSD) in 10,123 adolescents aged 13-18 years to be 5\% (and 7\% for those aged 17-18 years) (1). A 1999 literature review found this number ranged from 3.5-8.1\% for adolescents, compared with adult populations which exhibited figures as low as $0.8-1.2 \%$ (2). Adolescents are at a particularly high risk for experiencing traumatic events (3) and developing PTSD as a result of these experiences (4). This is especially worrisome because youth is a critical stage of development, and having PTSD can hinder maturation and identity formation and lead to life-long deficits with necessary mental and emotional skills (2).

Post-traumatic stress disorder is significantly associated with an increased risk for suicidal behavior $(5,6)$, though this association has been much less researched than the relationship between suicidal behavior and other disorders known to be risk factors, like major depression and substance-use disorders (7). One study found that among 3,021 adolescents and young adults, aged 14-24 years, who made past suicide attempts, PTSD corresponded with a higher risk for suicide attempts than any other psychiatric illness (8). 
According to the World Health Organization (WHO) (9), suicide is one of the top 3 causes of death among those aged 10-24 years. About 4 million adolescents attempt suicide every year and more than 100,000 of attempters successfully take their own life, resulting in one youth suicide every 5 minutes around the world (10). Annually, suicides are responsible for $1.6 \%$ of all deaths in the US, but $15.6 \%$ of deaths among those aged 15-24 years (11). Judging by the fact that suicide is extremely difficult to predict in the clinical setting (12), focusing a great deal of effort on preventing suicidal behavior outside of the clinical setting is of the utmost importance (e.g., limiting access to lethal means or implementing guidelines for the media to follow when reporting about suicides). The purpose of this review is to discuss and critically analyze prevention methods for suicidal behavior in adolescents with PTSD.

\subsection{PTSD and suicidal behavior in adolescents}

More than $90 \%$ of youth suicide victims have at least one diagnosable psychiatric disorder at the time of death (13). Seventy-five percent of those with PTSD have other mental health problems (14). Comorbidity of PTSD with other psychiatric conditions (like mood disorders, substance-use disorders, and psychosis) is associated with an increased suicide risk and higher rates of suicidal behavior (7). Depression is the most prevalent disorder among youths who have taken their own lives $(13,15)$. Taken together, this might lead one to consider a few of the (many) possibilities of causal interaction between PTSD and suicidal behavior; certain PTSD symptoms cause depression, which in turn leads to suicidal behavior or even that specific attributes or symptoms common to all or some of the disorders mentioned above (such as sleep disturbances) independently predict suicidal behavior (7). However, with so many variables and risk factors to take into account, the causal chain of events linking PTSD and suicidal behavior remains unclear. As a number of studies have pointed out, while almost all suicide victims have at least one psychiatric disorder, most patients who have only one or more psychiatric disorders (without any other risk factors) do not actually commit suicide (16). Thus, in mapping out possible avenues of causality between PTSD and suicide, all other risk factors for suicide that might play a role must be taken into account.

There is some evidence that PTSD can be an independent risk factor for suicidal behavior $(17,18)$. For example, a cohort study of 1,698 young adults who grew up in Baltimore revealed a significantly increased risk of suicide attempts for those who had PTSD compared to those without the disorder and those who experienced a traumatic event without developing the illness; and this increased risk remained unchanged even after adjusting for some other notable risk factors, like depression and sex (17). Research conducted by Mazza on 106 adolescents in grades 9-12 revealed that PTSD symptomology was significantly related to a heightened risk of suicidal ideation after adjusting for depression and gender (18). Unfortunately, these studies failed to 
control for a sufficient amount of associated risk factors, like childhood sexual abuse, in order to rule out the effects of confounding variables that were not statistically analyzed in these studies.

It is also possible that trauma and stressful experiences can lead to suicidality without a mediating PTSD diagnosis. Traumatic and stressful events, such as exposure to terrorism and physical and sexual abuse have all been found to be risk factors for PTSD, suicide, or other suicidal behaviors (19-21). However, the cohort study that was already described (17) found that only PTSD, and not traumatic experiences, independently predicted suicide attempts. Also of important note in this study was the finding that PTSD caused by trauma that involves assaultive violence was significantly associated with suicide attempts, whereas PTSD caused by trauma that did not involve assaultive violence was not significantly related to suicide attempts. This finding may suggest a differential association between suicidal behavior and the type of trauma experienced.

One way of assessing the association between suicide and PTSD is to focus on some of the specific symptoms of PTSD that might be risk factors for suicide. One study found that increased levels of hostility, depression, and arousal were related to higher suicide risk (22). Hostility and increased arousal fall within the hyperarousal cluster of PTSD symptoms. However, levels of avoidance (avoidance cluster) were negatively correlated with suicide risk, leading the authors to hypothesize that avoidance may serve to lessen the chances of suicide. In a longitudinal study, Sourander and colleagues looked at a group of 580 adolescents and found that those who exhibited aggressive behaviors (which fall into the hyperarousal cluster) at age 8 were significantly more likely to show suicidal behaviors (specifically, suicide attempts and suicidal thinking) 8 years later, at age 16 years (23). Impulsivity (associated with the hyperarousal cluster) has also been documented to have strong ties to both PTSD and suicidal behavior. For example, Kotler and colleagues found a positive association between impulsivity and risk of suicide in patients with PTSD, as compared with matched controls, who showed no association between these two factors (24). Given the fact that the majority of suicidal crises are short-lived, unplanned, and associated with ambivalent feelings of whether to live or to die (25), impulsivity seems to be an especially strong link between PTSD and suicide. The re-experiencing symptom cluster of PTSD has also been positively associated with PTSD and suicide (26). With regards to the efficacy of different coping strategies, Amir et al documented that in PTSD patients, psychological coping mechanisms like minimization, mapping, and replacement were associated with lower risk of suicide, and that suppression was associated with an increased risk of suicide (27).

Other risk factors for suicide include gender, a family history of suicide, and past suicide attempts. Men successfully complete suicide more often and tend to use highly lethal means, as compared with females who attempt more suicides using low-lethality procedures (20). A family history of suicide and previous suicide attempts are two of the most significant risk factors for suicide (28). A family history of suicide may increase the 
chance of developing PTSD (29) and previous suicide attempts are potentially traumatizing experiences in and of themselves. Having a family history of suicide or having a history of suicide attempts can also potentially lead to contagion-like effects.

Contagion, a phenomenon by which interpersonal contact with suicidal individuals results in the spread of suicidal behaviors (with suicide methods often being mimicked or imitated), sometimes throughout entire communities, is more common among youth than among those in other age groups $(13,29)$. A special type of contagion called the Werther effect refers to copycat suicides spurred on by media coverage of people taking their own lives. Media induced contagion effects will be discussed in detail later on.

In terms of neurobiological underpinnings, serotonergic abnormalities are a common finding in post-mortem analyses of the brains of suicide victims $(16,30)$. Compared with those who die of causes other than suicide, suicide victims are generally found to have lower levels of pre-synaptic serotonin transporter sites in various brain regions, including the hypothalamus, the brainstem, the prefrontal cortex, and especially in the ventral medial region of the prefrontal cortex $(31,32)$. Suicide victims also tend to have higher levels of 5-HT1A post-synaptic receptors, with this also being most evident in the ventral medial prefrontal cortex (31). The ventral medial prefrontal cortex is associated with executive functioning, so impairment of this brain region may result in deficits in the ability to inhibit distressing, aggressive, or suicidal emotions, and thus lead to more impulsivity $(16,30)$. As mentioned, impulsivity and aggression are strongly linked to both PTSD and suicidal behavior. Reduced levels of the principal metabolite of serotonin, 5-HIAA, in the cerebrospinal fluid have been consistently observed in both those who have committed suicide and those who have attempted suicide $(16,30,33)$. Amongst suicide attempters, 5-HIAA has been reported to be negatively correlated with the lethality of the suicide attempt (30). Decreased serotonergic activity has also been observed in victims of childhood abuse (16) and other traumatic experiences, as well as in patients with PTSD. One recent study (34) found that patients with PTSD had significantly reduced levels of serotonin 1B receptors as compared to those without PTSD. Furthermore, those who were exposed to traumatic experiences without developing PTSD also had lower levels of serotonin $1 \mathrm{~B}$ receptors (though levels were not as those with full blown PTSD). Perhaps the most important evidence for serotonergic abnormalities in PTSD patients is the relative effectiveness of selective serotonin reuptake inhibitors (SSRIs) in the treatment of this disorder (35). In terms of the prefrontal cortex, imaging studies have revealed lower levels of activation in the medial prefrontal region when patients with PTSD are presented with various stress-inducing stimuli $(36,37)$. These deficits may lead to some of the symptoms and behaviors associated with PTSD, like aggression, impulsivity, depression, heightened startle response, and suicidal behavior. Also, when taken alone, these symptoms and behaviors are indirect evidence of serotonergic impairments, as well as prefrontal cortex deficits (35). 
Trauma, PTSD, and suicide have all been associated with dysregulation of the hypothalamic-pituitary-adrenal (HPA) axis $(16,29)$. As suggested by animal models using maternal deprivation procedures, hyperactivity of the HPA axis (including physiological alterations like elevated glucocorticoid levels and increased sensitivity to stress) appears to be linked to traumatic experiences $(38,39)$. Surprisingly, PTSD is frequently associated with an increased inhibition of the HPA stress response and average or reduced levels of cortisol (40). However, as Yehuda explains, it is not necessarily the case that individuals with PTSD display lowered HPA and stress and stress responsiveness (40), as indicated by reports that increased cortisol and HPA responses are exhibited by individuals with PTSD who are exposed to reminders of traumatic events (in comparison with controls) (41, 42). Some of these contrasting findings might be explained by individual differences in genetic predisposition, the type of trauma experienced, or the age at which trauma is experienced. A much more consistent finding in the literature is a reduced hippocampal volume, which is considered a core feature of PTSD (29). Because memory, learning, and fear conditioning are highly dependent on the hippocampus, damage to this brain area may play a role in PTSD by causing traumatized individuals to over-generalize fear responses associated with their traumatic experiences to novel environments or people and exhibit hypervigilance and stressful reactions in even the safest of situations (29). Overactivity of the amygdala, as shown in patients with PTSD who are presented with stressinducing stimuli $(43,44)$, may also help to explain such fear and stress responses. Taken together, these deficits can cause symptoms related to both PTSD and suicide, such as helplessness, aggression, negative expectations, low self-esteem, and selfdestructiveness $(16,29)$. The HPA axis also has connections with the sympathetic nervous system via the hypothalamus, where fear signals are passed along. Overactivity of the sympathetic nervous system, as evidenced by heightened norepinephrine responsiveness to perceived threat, and elevated heart rate and blood pressure, is also associated with the hyperactivity of the stress response (35). Furthermore, abnormalities in neurotransmitter systems associated with controlling and mediating the stress response such as the neuropeptide Y, endogenous opioid, gammaaminobutyric acid (GABA), and glutamate systems have also been linked to PTSD and the dysregulated stress reaction associated with sensitization (29).

Finally, it may be the case that PTSD, suicide, and other associated conditions all share a similar genetic, epigenetic, and/or neurobiological predisposition $(16,29)$. For example, genetic and neurobiological differences associated with sex may play a role in predisposing someone to PTSD and suicidal behavior. As previously mentioned, women commit suicide less often than men do. Women have been reported to exhibit higher levels of 5-HIAA in the cerebrospinal fluid as compared to men (45). Thus, sex may represent a predisposition towards higher rates of both suicide and PTSD in men because these disorders are associated with hypoactvity of serotonergic functioning. What seems most likely is that these disorders are caused by a mixture of 
both a shared underlying predisposition and deficits acquired after the development of PTSD (or other common comorbid conditions).

\subsection{Prevention}

There has been a good deal of research conducted on suicide prevention for the general population and also some dealing with suicide prevention in adolescent populations. However, there have been no empirical investigations of prevention methods tailored to suicidal adolescents with PTSD.

There are two general options when it comes to suicide prevention: decreasing risk factors for suicide or case finding, which refers to looking for and identifying at risk persons for referral and treatment (5). A literature review by Mann et al found that the three most effective prevention techniques have proven to be means restriction, education of primary care physicians, and gatekeeper training (46).

\subsubsection{Means restriction}

Means restriction refers to any measures taken to decrease access to or availability of suicide means (24). Suicide attempters tend to use specific, favored methods, and when they are unable to attain the means for these favored methods, they are less likely to make a suicide attempt (47).

The main justification for implementing means restriction measures is that the majority of suicidal crises and feelings of wanting to kill oneself are brief and transient, unplanned or impulsive, and often accompanied by mixed feelings of whether to live or to die (24). Thus, with more lethal means unavailable or inaccessible during this short window of opportunity, the suicidal person will either not go through with the attempt or use less lethal means (24). Given the evidence that impulsivity may serve as a strong link between PTSD and suicide, means restriction seems as if it might be just as effective if a suicidal crisis is spurred on by PTSD-related impulsivity or other PTSD symptoms associated with suicide.

According to the Centers for Disease Control and Prevention (CDC), the top three methods used by young people in suicides are firearms (45\%), suffocation (40\%), and poisoning (8\%) (48). One study found that across US, suicide rates are higher for people living in locations where there are more guns in homes and that this association is most prominent for adolescents aged 5-19 years (49). In support of means restriction, studies consistently show that passing firearm control laws lead to reductions in suicide rates $(50,51)$. For example, the passing of gun control legislation in 1976 in the District of Columbia led to a 23\% reduction in firearm-related suicides (50). Similarly, gun control laws in South Australia led to significant decreases in suicides using firearms, in comparison with other Australian states without similar gun 
laws in place (where there was an increase suicide rates) (51). For suicidal adolescents with PTSD, one potentially beneficial future avenue for research might be to focus on figuring out if PTSD caused by certain types of trauma leads to using specific suicide means or methods more often than others. For example, it seems reasonable to think that those who experience gun-related trauma might be less likely to choose firearms as their means because they will probably seek to avoid any reminder of the trauma at the risk of triggering re-experiencing symptoms. The finding that avoidance behaviors are negatively correlated with suicide risk (22), might provide some support for this hypothesis.

\subsubsection{Physician education and training}

One of the most promising prevention methods of suicide in adolescents is to educate and train primary care physicians to better screen, identify, and treat patients with psychiatric disorders, as well as to refer such patients to mental health care professionals (46). However, an excessive amount of physicians fail to identify and effectively give much needed support or treatment to patients with suicidal inclinations and associated psychiatric disorders, like depression (15). This failure to recognize depression and other risk factors related to suicide may be a major reason why most suicide victims go untreated before taking their own lives (46). Physician education may be of particular importance in children and youth because it is often the case that physicians are a youth's only source of mental health care service (52). The prevention of suicide in the primary care setting can potentially be an extremely effective method because about $75 \%$ of all victims have contact with a physician within a year of suicide and almost half of all victims have contact with a physician within a month of taking their own lives (53). In one study based in Australia, primary care physicians took part in a training workshop that was intended to help them to better identify and effectively respond to young people at risk for suicide. Despite the fact that the workshop only lasted for one day, identification of suicidal patients increased by $130 \%$ (54). Other studies also show that this prevention method leads to reductions in suicide rates and increases in rates of identifying those at an elevated risk for suicide $(55,56)$.

Physicians should screen all adolescents to assess coping skills and any thoughts or behaviors, past and present, which are associated with suicidal behaviors (57). With regards to helping suicidal adolescents with PTSD, physicians should also be adept in screening for, identifying, and triaging adolescents with PTSD (and other disorders related to increased risk of suicide). The Society for General Internal Medicine enlisted the help of the American Association of Medicine and Psychiatry, the American Psychiatric Association, The American College of Physicians, and the American Academy of Physician in order to provide physicians with literature and guidelines to effectively diagnose and treat psychiatric disorders commonly 
observed in primary care (58). Furthermore, physicians might also want to assess which type of trauma led to PTSD for different individuals so as to understand avoidance patterns and to get a grasp on which suicide methods might be most favorable for each individual. Taking screening and assessment results into account, the clinician can also serve to form a support system for the youth patients by opening lines of communication with parents and other adults and potential gatekeepers in the local community, including teachers, activity partners, etc. (59). In general, by forming respectful, encouraging, and most importantly, understanding relationships with adolescent patients, primary care physicians can open a much needed line of support for adolescents in times of crisis or distress (59), which would be especially useful for those suicidal youth with comorbid or particularly distressing disorders, like PTSD and depression.

Screening instruments, which may focus on risk factors for suicide or suicide alone (46), are valuable tools that physicians should utilize. These instruments reliably lead to accurate recognition of and significantly higher detection rates of at-risk youth populations $(60,61)$.

\subsubsection{Mental health care}

Of all of the many varieties of therapies that are typically utilized in an attempt to treat suicidal people, dialectical behavioral therapy is the only one that has continuously proven to be effective in lessening suicidal behavior in the adult population (20). Unfortunately, none of the research found on this topic has focused any attention specifically on suicidal behavior in adolescents with PTSD. With specific regards to coping strategies, clinicians should teach and encourage PTSD patients to use coping strategies associated with lower risk of suicide in PTSD patients, like replacement, mapping, and minimization, and to avoid those associated with higher risk of suicide in PTSD patients, like suppression (25). According to Sher and Ganz, a combination of pharmacotherapy and psychotherapy is likely to be the best treatment option for mental health professionals to give to adolescents with PTSD (29).

\subsubsection{Gatekeeper training}

Gatekeeper training refers to educating and training adult community members who communicate and interact with adolescent populations on a day-to-day basis to recognize youth at risk for suicide, assess their level of risk, and refer them to mental health professionals for treatment when necessary (46). Examples of potential gatekeepers are teachers, guidance counselors, coaches, prison and military officials, clergy and other religious professionals, police, first responders, nurses, primary 
care physicians, and social workers $(46,62)$. Unfortunately, many potential gatekeepers lack either knowledge of the risk factors for youth suicide or do not possess the appropriate attitudes or intervention skills to effectively take action when needed. For example, a nationwide survey of US high school teachers showed that fewer than $10 \%$ of all teachers felt that they could identify at-risk students, and even amongst counselors who possessed knowledge of the risk factors; two thirds did not think that they could recognize students at risk for suicide (63). It becomes even more important to contrive gatekeeper strategies when one takes into account a finding by Wyman and colleagues: of a mixed sample of 2,059 8th and 10th grade students surveyed with regards to help-seeking attitudes, those who mentioned a past suicide attempt were much less likely than those without a past suicide attempt to seek help or believe that any school personnel could help them (64).

The cumulative literature on this topic has not yet proven gatekeeper training to have an independent or significant effect on diminishing suicidal behavior (62). However, there have been some reports that point towards positive effects of these training programs.

A study based in Quebec (65) gave gatekeeper training to 43 adult "helpers" who worked with youth populations. Within 6 months of the training date, over $60 \%$ of the helpers made an intervention with a suicidal youth, and the adults given the training attained increased intervention skills and knowledge of suicidal behavior as compared with 28 helpers in a control group who didn’t receive the training.

\subsubsection{Public education programs}

Public education programs attempt to provide suicide awareness information and inform the public about risk factors and identification strategies, as well as to help cultivate appropriate attitudes towards suicide and suicidal behavior. These programs are often targeted at adolescent populations (13) and especially in the school setting (28). In one study, a school-based education campaign was utilized. Adolescents participated in meetings that focused on opening them up to inner conflicts and negative life experiences associated with suicidal behavior, as well as promoting positive coping mechanisms to help them to deal with distressing feelings and emotions. Results showed that because of the intervention, students were able to cope more effectively with stress and negative feelings like hopelessness (66). Hopelessness is a symptom associated with both PTSD and suicidal behavior in adolescents. However, there is not enough empirical evidence investigating the efficacy of this method of suicide prevention to make any definite conclusions or recommendations $(28,46)$. Such preventative interventions can also aim to target more specific at-risk populations like suicidal adolescents with PTSD by including information about identifying individuals in this population, risk factors, and treatment options. 


\subsubsection{Media guidelines}

As evidenced in the literature, the media (print, television, internet) can help to facilitate reductions of youth suicide by providing supportive advice and educating the public or can have potentially disastrous outcomes, invoking mass contagion effects as a result of irresponsible coverage of suicides (e.g., romanticizing or glamorizing suicide, or dramatizing more lethal suicide methods) (24, 46, 67-69). Improper coverage can even lead to the teaching of new or alternative suicide methods (24), as well as draw attention to suicide hot spots (70). In this context, the effects of contagion can be far more calamitous than contagion via person-person contact in community and local settings (as already discussed) (67). Furthermore, there is good evidence that youth populations are at the greatest risk to media-related contagion effects $(68,71)$ : Phillips and Carstensen $(68)$ reported that TV news and feature stories about suicide caused significant increases in teenage suicide over the week following these broadcasts (almost a 7\% increase) compared with adult suicides that increased by less than half of a percent. In addition, broader coverage of these stories by more news stations led to higher numbers of teen suicide. It should be noted that because this study took place in the US, the generalizability of these results to other countries (where the impact of TV news might be different) is unknown.

Media contagion (also commonly referred to as the "Werther effect") can in large part be explained by social learning theory (67). In the case of the Werther effect, greater public attention can serve as a reward to the observer and can present as longer duration of coverage, greater amounts of coverage (67), and greater prominence of coverage, including front page placement or covering celebrity suicides (72). Furthermore, observers are more likely to model behavior off of others that they share similar characteristics with (like age or gender) and maintain more attention on viewing stories that involve such people $(73,74)$. For example, for upto 70 days after the showing of a weekly serial that depicted the railway suicide of a 19-year-old male student, the number of railway suicides was highest amongst the population of 15-19-year-old males (with rates increasing by upto 175\%), as compared to all other age/gender populations (74).

There are also a few studies looking into the potential relationship between PTSD and media effects. Researchers dealing with 69 youths in the sixth grade who lived near Oklahoma City when the 1995 bombing took place, but were not directly exposed to the event, found that indirectly witnessing the event via media sources was significantly associated with acquiring PTSD symptoms (75). Another study based in Ontario studying 143 undergraduate students (aged 17-37 years), looked at the PTSD symptomology related to media exposure to the $9 / 11$ terrorist attacks. Media exposure was significantly associated with two of the three PTSD symptom clusters, including hyperarousal and re-experiencing symptoms, but was not associated with the avoidance cluster (76). Taken in light of results already mentioned, that PTSD symptoms like hyperarousal 
and re-experiencing symptoms in youth lead to an increased risk of suicide, it can be inferred that media coverage of certain events can serve to heighten these risk factors even more and create an even shorter bridge between PTSD and suicide.

As already briefly mentioned if conducted in a responsible manner, media reports on suicide can have potentially beneficial effects (the Papageno effect). The main prevention method for suicidal contagion and other adverse effects of the media is providing reporting guidelines for media organizations to follow $(46,77)$. Such guidelines may include avoidance of sensationalizing or dramatizing suicides, encouraging suicidal people to seek treatment and care, and educating them about the risk factors and effective coping mechanisms, educating those involved in the production and reporting about contagion, avoidance of elaborating on specifics of suicide methods or hot-spots, and limiting the coverage and prominence of suicide cases and avoiding front page placement (67). These guidelines listed are some that help to form a set of national guidelines developed by the American Foundation for Suicide Prevention (78). The Centers for Disease Control and Prevention has also released similar guidelines for media reporting (79). Although no research has focused on the efficacy of these specific guidelines $(46,67)$, there has been some work done on others. For example, in Vienna, Austria suicide rates, and in particular, rates of subway suicides, increased dramatically after the subway system was put into place in 1978. Elmar and Sonneck documented an $80 \%$ decrease in subway suicides after guidelines were developed by the Austrian Association for Suicide Prevention and spread via a media campaign in 1987 (80). In another study, after Swiss newspapers and magazines implemented a set of guidelines, researchers found that in comparison to before the guidelines, articles received less prominent placement, were not as lengthy, less sensational, and included less pictures (81). Taking into account the studies on media coverage of PTSD, similar guidelines can be put into place with minor additions to account for the risk factors and symptoms associated with suicidal adolescents with PTSD.

There have been relatively few research efforts that have looked to see if the Internet might have a relationship with suicidality (82). However, the internet is of particular concern in light of the facts that social networking sites can be utilized to spread information in just minutes (24), there are suicide chat rooms and websites containing instructions for different suicide methods (46), and almost all young Americans and adolescents have Internet access to one degree or another (with 93\% of all 12-17 year olds and 89\% of 18-24 year olds being online in 2009) (83).

The most used social media site, Facebook, reached 500 million registered users in 2010 (84) and in 2009, in general, social networking sites were used on a regular basis by $73 \%$ of teens, including $55 \%$ of $12-13$ year olds and $82 \%$ of $14-17$ year olds ( 85 ). There is evidence that Facebook and other social media sites may actually serve to increase social support and boost mental well-being (86) and even stop or decrease suicidal ideation in young people and adolescents (82). One especially worrisome online venue is websites devoted to promoting suicide by detailing information on 
suicide means and methods, of which there were found to be more than 100,000 in 1999 (87). These websites suggest suicide as a solution to life's issues and contain detailed descriptions of methods yielding the maximum effect, as well as suicide notes and pictures of people who committed suicide. They exert group pressure to fulfill suicide plans, glorifying those who have committed suicide. Other potential avenues for obtaining information about suicide include forums and self-help pages, in which other users can leave comments or feedback. In 2010, Ries (88) documented a case in which a 24-year-old male posted a comment on a forum about the intention to kill himself. While some showed support and sympathy, others egged him on. The young man went on to kill himself. In terms of Internet-related PTSD effects, one study found that amongst elementary school children who indirectly witnessed or heard about the 9/11 terrorist attacks via different media outlets, children who saw or heard about it on the Internet reported more PTSD symptoms than those who witnessed the event by way of television or print media (89).

A recent cohort study (82) conducted with 719 14-24 year olds found that 59\% of participants saw or learned about suicide stories on the Internet. Mental health information was also attained from the participants. Although hopelessness and knowing someone who attempted or committed suicide at baseline were both highly related to learning about suicides from social networking sites, social media was not related to increases in suicidal ideation in these groups, suggesting that these sites may actually serve to be effective mediums for social support and preventing suicidal behavior. The same beneficial effects were not observed with online forums, which were related to increases in suicidal ideation, providing evidence that forums may serve to encourage or facilitate suicidal behavior. The same goes for blogging, which was related to hopelessness. These results may indicate that youths with PTSD or other mental health problems associated with suicide may be best off visiting social media sites as opposed to forums or blogs.

Like with other types of media prevention, the Internet can also be a medium used to adequately educate adolescents about suicide, its causes, risk factors, and treatment options. In order to combat the deleterious effects of suicide-promotion sites and other harmful venues, suicide-prevention websites can be utilized to support and guide suicidal youth to teach effective coping strategies and proper treatment options (82). Furthermore, mental health providers and gatekeepers like primary care physicians and teachers can help educate youth of the dangers and benefits of Internet use. Also, as already discussed, Facebook and other social media sites may actually help to prevent suicide by allowing support and guidance from friends and other people in one's social network $(13,86)$. Finally, media guidelines for reporting suicide on the Internet have been put together, but thus far no research investigations have studied the effects of such guidelines $(46,78,90)$. All of the fore mentioned prevention options could be utilized for suicidal adolescents with PTSD and/or other psychiatric conditions. 


\subsubsection{Multi-dimensional prevention methods}

Given the demonstrated efficacy of most of the prevention methods discussed thus far, it seems reasonable to hypothesize that a more versatile, multi-dimensional suicide prevention method, combining all or some of these methods, might prove to have the best results of all. In a study based in Nuremberg, Germany (91) both primary care physicians and community gatekeepers (clergy, help-lines, mental health care workers, policemen, prison officers) were given suicide prevention training. Furthermore, media organizations (TV, radio, and newspaper) were given guidelines to follow concerning reporting of suicides in order to prevent copycat suicides and contagion-related effects. There was also a public education campaign launched which organized lectures for the general public and enlisted the help of public officials and various media outlets (including a cinema trailer and an internet website) to help educate community members and health care workers about depression (which of course, is one of the main risk factors for suicide) and to encourage people with depression or depressive symptoms to seek help. Results showed that over a 2-year period, there was a $24 \%$ reduction in suicidal acts (which included completed suicides and suicide attempts), and that this reduction was significant compared with the control region (Wuerzburg, Germany) that did not receive the prevention programs. In Wuerzburg, suicidal acts increased by over 7\% over the 2-year span. More research needs to be conducted on such multi-dimensional approaches in order to come to any concrete conclusions about the efficacy of this method compared with the other methods discussed.

\section{References}

1. Merikangas KR, He J-P, Burstein M, Swanson SA, Avenevoli S, Cui L, et al. Lifetime prevalence of mental disorders in US adolescents: results from the National Comorbidity Study-Adolescent Supplement (NCS-A). J Am Acad Child Adolesc Psychiatry 2010;49(10):980-9.

2. Deykin EY. Posttraumatic stress disorder in children and adolescence: a review. Medscape Psychiatry and Mental Health eJournal 1999;4(4). URL: http://www.westga.edu/ vickir/Healthcare/ HC07\%20PTSDPrimer/Link\%207\%20--\%20PTSD\%20in\%20Children\%20\&\%20Adolescents.pdf. Accessed 01 October, 2012.

3. Breslau N, Kessler RC, Chilcoat HD, Schultz LR, Davis GC, Andreski P. Trauma and posttraumatic stress disorder in the community: the 1996 Detroit Area Survey of trauma. Arch Gen Psychiatry 1998;55(7):626-32.

4. Costello EJ, Erkanli A, Fairbank JA, Angold A. The prevalence of potentially traumatic events in childhood and adolescence. J Trauma Stress 2002;15(2):99-112.

5. Sareen J, Houlahan T, Cox BJ, Asmundson GJG. Anxiety disorders associated with suicidal ideation and suicide attempts in the National Comorbidity Survey. J Nerv Ment Dis 2005;193:450-4.

6. Kessler RC, Sonnega A, Bromet E, Hughes M, Nelson C. Posttraumatic stress disorder in the National Comorbidity Survey. Arch Gen Psychiatry 1995;52:1048-60.

7. Panagioti M, Gooding P, Tarrier M. Post-traumatic stress disorder and suicidal behavior: a narrative review. Clin Psychol Rev 2009;(6):471-82. 
8. Wunderlich U, Bronisch T, Wittchen HU. Comorbidity patterns in adolescents and young adults with suicide attempts. Eur Arch Psychiatry Clin Neurosci 1998;248(2):87-95.

9. World Health Organization. Suicide prevention (SUPRE), 2012. URL: http://www.who.int/mental_ health/prevention/suicide/suicideprevent/en/. Accessed 01 October, 2012.

10. World Health Organization. The second decade: improving adolescent health and development, 1998. URL: http://www.who.int/mip2001/files/2281/2ndDecadefulldocument.PDF. Accessed 01 October, 2012.

11. American Association of Suicidology. USA suicide: Official financial data, 2010. URL: http://www. suicidology.org/c/document_library/get_file?folderld=248\&name=DLFE-618.pdf. Accessed 01 October, 2012

12. Sher L. Preventing suicide. Q J Med 2004;97:677-80.

13. Gould MS, Greenberg T, Velting DM. Youth suicide risk and preventive interventions: a review of the past 10 years. J Am Acad Child Adolesc Psychiatry 2003;42(4):386-405.

14. Kataoka S, Langley AK, Wong M, Baweja S, Stein B. Responding to students with PTSD in schools. Child Adolesc Psychiatr Clin N Am 2012;21(1):119-33.

15. Lake CR. How academic psychiatry can better prepare students for their future patients. Part I: the failure to recognize depression and risk for suicide in primary care; problem identification, responsibility, and solutions. Behav Med 2008;34(3):95-100.

16. Mann JJ. Neurobiology of suicidal behavior. Nat Rev Neurosci 2003;4:819-28.

17. Wilcox H, Storr CL, Breslau N. Posttraumatic stress disorder and suicide attempts in a community sample of urban American young adults. Arch Gen Psychiatry 2009;66(3):305-11.

18. Mazza JJ. The relationship between posttraumatic stress symptomatology and suicidal behavior in school-based adolescents. Suicide Life Threat Behav 2000;30(2):91-103.

19. Johnson JG, Cohen P, Gould MS, Kasen S, Brown J, Brook JS. Childhood adversities, interpersonal difficulties, and risk for suicide attempts during late adolescence and early adulthood. Arch Gen Psychiatry 2002;59(8):741-9.

20. Mazza, JJ. Youth suicidal behavior: a crisis in need of attention. Adolesc Ment Health 2006:156-77.

21. Chemtob CM, Horenczyk RP, Madan A, Ptiman SR, Wang Y, Doppelt O, et al. Israeli adolescents with ongoing exposure to terrorism: suicidal ideation, posttraumatic stress disorder, and functional impairment. J Trauma Stress 2011;24(6):756-9.

22. Ben-Ya'acov Y, Amir M. Posttraumatic symptoms and suicide risk. Pers Individ Diff 2004;36: 1257-64.

23. Sourander A, Helstela L, Haavisto A, Bergroth L. Suicidal thoughts and attempts among adolescents: a longitudinal 8-year follow-up study. J Affect Disord 2001;63(1-3):59-66.

24. Kotler M, lancu I, Efroni R, Amir M. Anger, impulsivity, social support, and suicide risk in patients with posttraumatic stress disorder. J Nerv Ment Dis 2001;189(3):162-7.

25. Yip PFS, Caine E, Yousuf S, Chang S-S, Wu KC-C, Chen Y-Y. Means restriction for suicide prevention. Lancet 2012;379:2393-9.

26. Bell JB, Nye EC. Specific symptoms predict suicidal ideation in Vietnam combat veterans with chronic post-traumatic stress disorder. Mil Med 2007;172(11):1144-7.

27. Amir M, Kaplan Z, Efroni R, Kotler M. Suicide risk and coping styles in posttraumatic stress disorder patients. Psychother Psychosom 1999;68(2):76-81.

28. Kuthcer SP, Szumilas M. Youth suicide prevention. Can Med Assoc J 2008;178(3):282-5.

29. Ganz D, Sher L. Terror and suicidal behavior in adolescents with posttraumatic stress disorder. Minerva Pediatr 2010;62(4):363-70.

30. Mann JJ, Oquendo M, Underwood MD, Arango V. The neurobiology of suicide risk: a review for the clinician. J Clin Psychiatry 1999;60(2):7-11.

31. Arango V, Underwood MD, Gubbi AV, Mann JJ. Localized alterations in pre and postsynaptic serotonin binding sites in the ventrolateral prefrontal cortex of suicide victims. Brain Res 1995;688(1-2):121-33. 
32. Mann JJ, Henteleff RA, Lagattuta TF, Perper JA, Li S, Arango V. Lower 3H-paroxetine binding in cerebral cortex of suicide victims is partly due to fewer high affinity, non-transporter sites. J Neural Transm 1996;103(11):1337-50.

33. Mann JJ, Arango V, Marzuk PM, Theccanat S, Reis DJ. Evidence for the 5-HT hypothesis of suicide. A review of post-mortem studies. Br J Psychiatry Suppl 1989;(8):7-14.

34. Murrough JW, Czermak C, Henry S, Nabulsi N, Gallezot D, Gueorguieva R, et al. The effect of early trauma exposure on serotonin type 1B Receptor expression revealed by reduced selective radioligand binding. Arch Gen Psychiatry 2011;68(9):892-900.

35. Heim C, Nemeroff CB. Neurobiology of posttraumatic stress disorder. CNS Spectr 2009;14(Suppl1): 13-24.

36. Shin LM, Orr SP, Carson MA, Rauch SL, Macklin ML, Lasko NB, et al. Regional cerebral blood flow in the amygdala and medial prefrontal cortex during traumatic imagery in male and female Vietnam veterans with PTSD. Arch Gen Psychiatry 2004;61(2):168-76.

37. Shin LM, Wright Cl, Cannistraro PA, Wedig MM, McMullin K, Martis B, et al. A functional magnetic resonance imaging study of amygdala and medial prefrontal cortex responses to overtly presented fearful faces in posttraumatic stress disorder. Arch Gen Psychiatry 2005;62(3):273-81.

38. Plotsky PM and Meaney MJ. Early, postnatal experience alters hypothalamic corticotropinreleasing factor (CRF) mRNA, median eminence CRF content and stress-induced release in adult rats. Brain Res Mol Brain Res 1993;18(3):195-200.

39. Rentesi G, Antoniou K, Marseios M, Fotopoulos A, Alboycharali J, Konstandi M. Long-term consequences of early maternal deprivation in serotonergic activity and HPA function in adult rat. Neurosci Lett 2010;480(1):7-11.

40. Yehuda R. Status of glucocorticoid alterations in post-traumatic stress disorder. Ann N Y Acad Sci 2009;1179:56-9.

41. Elzinga BM, Schmahl CG, Vermetten E, van Dyck R, Bremmer JD. Higher cortisol levels following exposure to traumatic reminders in abuse-related PTSD. Neuropsychopharmacology 2003; 28(9):1656-65.

42. Liberzon I, Abelson JL, Flagel SB, Raz J, Young EA. Neuroendocrine and psychophysiologic responses in PTSD: a symptom provocation study. Neuropsychopharmacology 1999;21(1):40-50.

43. Rauch SL, van der Kolk BA, Fisler RE, Alpert NM, Orr SP, Savage CR, et al. A symptom provocation study of posttraumatic stress disorder using positron emission tomography and script-driven imagery. Arch Gen Psychiatry 1996;53(5):380-7.

44. Liberzon I, Taylor SF, Amdur R, Jung TD, Chamberlain KR, Minoshima S, et al. Brain activation in PTSD in response to trauma-related stimuli. Biol Psychiatry 1999;45(7):817-26.

45. McBride PA, Tierney H, DeMeo M, Chen JS, Mann JJ. Effects of age and gender on CNS serotonergic responsivity in normal adults. Biol Psychiatry 1990;27(10):1143-55.

46. Mann JJ, Apter A, Bertolote J, Beautrais A, Currier D, Haas A, et al. Suicide prevention strategies: a systematic review. J Am Med Assoc 2005;294(16):2064-74.

47. Griffith EH. Suicide: closing the exits. J Am Med Assoc 1990;263(22):3091-2.

48. Centers for Disease Control and Prevention. Suicide prevention. URL: http://www.cdc.gov/ violenceprevention/pub/youth_suicide.html. Accessed 01 October, 2012.

49. Miller M, Lippmann SJ, Azrael D, Hemenway D. Household firearm ownership and rates of suicide across the 50 United States. J Trauma 2007;62(4):1029-35.

50. Loftin C, McDowall D, Wiersema, B, Cottey TJ. Effects of restrictive licensing of handguns on homicide and suicide in the District of Columbia. N Engl J Med 1991;325:1615-20.

51. Snowdon J, Harris L. Firearms suicides in Australia. Med J Aust 1992;156(2):79-83.

52. Horwitz SM, Kelleher K, Boyce T, Jensen P, Murphy M, Perrin E, et al. Barriers to health care research for children and youth with psychosocial problems. J Am Med Assoc 2002;288(12):1508-12.

53. Luoma JB, Martin CE, Pearson JL. Contact with mental health and primary care providers before suicide: a review of the evidence. Am J Psychiatry 2002;159(6):909-16. 
54. Pfaff JJ, Acres JG, McKelvey RS. Training general practitioners to recognise and respond to psychological distress and suicidal ideation in young people. Med J Aust 2001;174(5):222-6.

55. Rutz W, von Knorring L, Wålinder J. Long-term effects of an educational program for general practitioners given by the Swedish Committee for the Prevention and Treatment of Depression. Acta Psychiatry Scand 1992;85(1):83-8.

56. Hannaford PC, Thomson C, Simpson M. Evaluation of an educational programme to improve the recognition of psychological illness by general practitioners. Br J Gen Pract 1996;46(407):333-7.

57. Duke NN, Boroswki I. Suicidal events in adolescents: how clear are the warning signs? Ped Health 2009;3(6):551-63.

58. Cole SA, Sullivan M, Kathol R, Warshaw C. A model curriculum for mental disorders and behavioral problems in primary care. Gen Hosp Psychiatry 1995;17(1):13-8.

59. Taliaferro L, Oberstar J, Borowsky IW. Prevention of youth suicide: the role of the primary care physician. J Clinical Outcomes Manag 2012;19(6):270-85.

60. Shaffer D, Scott M, Wilcox H, Maslow C, Hicks R, Lucas CP, et al. The Columbia Suicide Screen: validity and reliability of a screen for youth suicide and depression. J Am Acad Child Adolesc Psychiatry 2004;43(1):71-9.

61. Joiner TE Jr, Pfaff JJ, Acres JG. A brief screening tool for suicidal symptoms in adolescents and young adults in general health settings: reliability and validity data from the Australian National General Practice Youth Suicide Prevention Project. Behav Res Ther 2002;40(4):471-81.

62. Isaac M, Elias B, Katz LY, Belik S, Deane FP. Gatekeeper training as a preventative intervention for suicide: a systematic review. Can J Psychiatry 2009;54(4):260-8.

63. King KA, Price JH, Telijohann SK, Wah J. High school teachers' perceived efficacy in identifying students at risk for suicide. J Sch Health 1999;69(5):202-7.

64. Wyman PA, Brown CH, Inman J, Cross W, Schmeelk-Cone K, Guo J, et al. Randomized trial of a gatekeeper program for suicide prevention: 1-year impact on secondary school staff. J Consult Clin Psychol 2008;76(1):104-15.

65. Chagnon F, Houle J, Marcoux I, Renaud J. Control-group study of an intervention training program for youth suicide prevention. Suicide Life Threat Behav 2007;37(2):135-44.

66. Orbach I, Bar-Joseph $\mathrm{H}$. The impact of a suicide prevention program for adolescents on suicidal tendencies, hopelessness, ego identity, and coping. Suicide Life Threat Behav 1993;23(2):120-9.

67. Gould MS. Suicide and the media. Ann NY Acad Sci 2001;932:200-21.

68. Phillips DP, Carstensen LL. Clustering of teenage suicides after television news stories about suicide. N Engl J Med 1986;315(11):685-9.

69. Sisask M, Varnik A. Media roles in suicide prevention: a systematic review. Int J Environ Res Public Health 2012;9(1):123-38.

70. Pirkis J, Blood RW. Suicide and the media. Part I: reportage in nonfictional media. Crisis 2001;22(4):146-54.

71. Gould MS, Jamieson P, Romer D. Media contagion and suicide among the young. Am Behav Sci 2003;46(9):1269-84.

72. Stack S. Media impacts on suicide: a quantitative review of 293 findings. Soc Sci Q 2000;81(4):957-71.

73. Stack S. Social correlates of suicide by age: Media impacts. In: Linaars AA, ed. Life Span Perspectives of Suicide: Time-lines in the Suicide Process. New York: Plenum, 1991:187-213.

74. Schmidtke A, Hafner $H$. The werther effect after television films: new evidence for an old hypothesis. Psychol Med 2009;18(3):665-76.

75. Pfefferbaum B, Seale TW, McDonald NB, Brandt EN Jr, Rainwater SM, Maynard BT, et al. Posttraumatic stress two years after the Oklahoma City bombing in youths geographically distant from the explosion. Psychiatry 2000;63(4):358-70.

76. Collimore KC, McCabe RE, Carleton RN, Asmundson GJ. Media exposure and dimensions of anxiety sensitivity: differential associations with PTSD symptom clusters. J Anxiety Disord 2008;22(6):1021-8. 
77. Ramadas S, Kuttichira P. The development of guidelines and its impact on the media reporting of suicide. Indian J Psychiatry 2011;53(3):224-8.

78. The American Federation for Suicide Prevention. Recommendations for reporting on suicide. URL: http://www.afsp.org/files/Misc_/recommendations.pdf. Accessed 01 October, 2012.

79. O'Carroll PW, Potter LB. Suicide contagion and the reporting of suicide: recommendations from a national workshop. MMWR Recomm Rep 1994;43(RR-6):9-17.

80. Etzersdorfer E, Sonneck G. Preventing suicide by influencing mass-media reporting. The Viennese experience 1980-1996. Arch Suicide Res 1998;4:64-78.

81. Michel K, Frey C, Wyss K, Valach L. An exercise in improving suicide reporting in print media. Crisis 2000;21(2):71-9.

82. Dunlop SM, More E, Romer D. Where do youth learn about suicides on the Internet, and what influence does this have to suicidal ideation? J Child Psychol Psychiatry 2011;52(10):1073-80.

83. Jones S, Fox S. Generations online in 2009. URL: http://www.pewinternet.org/ /media//Files/ Reports/2009/PIP_Generations_2009.pdf. Accessed 01 October, 2012.

84. Zuckerberg M. 500 million stories, 2010. URL: http://blog.facebook.com/blog.php?post= 409753352130. Accessed 01 October, 2012.

85. Lenhart A, Purcell K, Smith A, Zickuhr K. Social media and mobile Internet use among teens and young adults. 2010. URL: http://web.pewinternet.org/ /media/Files/Reports/2010/PIP_Social_ Media_and_Young_Adults_Report_Final_with_toplines.pdf. Accessed 01 October, 2012.

86. Ellison NB, Steinfeld C, Lampe C. The benefits of Facebook "friends:" Social capital and college students' use of online social network sites. J Comput Mediat Commun 2007;12(4):1143-68.

87. Dobson R. Internet sites may encourage suicide. Br Med J 1999;319(7206):337.

88. Ries B. Anatomy of an Internet suicide, 2010. URL: http://www.thedailybeast.com/articles/2010/ 08/31/reddit-suicide-how-the-internet-can-help-and-hurt.html. Accessed 01 October, 2012.

89. Saylor CF, Cowart BL, Lipovsky JA, Jackson C, Finch Jr AJ. Media exposure to September 11: elementary school students' experiences and posttraumatic symptoms. Am Behav Sci 2003;46(12):1622-42.

90. Ganz D, Braquehais MD, Sher L. Secondary prevention of suicide. PLoS Med 2010;7(6):e1000271.

91. Hegerl U, Althaus D, Schmidtke A, Niklewski G. The alliance against depression: 2-year evaluation of a community-based intervention to reduce suicidality. Psychol Med 2006;36(9):1225-33. 
